\title{
Rotating Day and Night Disturb Growth Hormone Secretion Profiles, Body Energy Metabolism, and Insulin Levels in Mice
}

\author{
Weihao Wang ${ }^{a, b}, c$ Zhengxiang Huang ${ }^{b}$ Lili Huang ${ }^{b}$ Chunlu Tan ${ }^{b}$ \\ Wanlin Chen ${ }^{b}$ Ferdinand Roelfsema ${ }^{d}$ Chen Chen ${ }^{b}$ Lixin Guo ${ }^{a, c}$ \\ ${ }^{a}$ Graduate School of Peking Union Medical College, Chinese Academy of Medical Sciences, Beijing, PR China; \\ ${ }^{b}$ School of Biomedical Sciences, University of Queensland, St Lucia, Brisbane, QLD, Australia; 'Department of \\ Endocrinology, Beijing Hospital, National Center of Gerontology, Institute of Geriatric Medicine, Chinese Academy

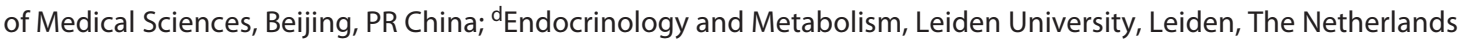

\section{Keywords}

Rotating light · Growth hormone secretion · Peripheral clock · Energy metabolism

\begin{abstract}
Background: Insulin and growth hormone (GH) - 2 vital metabolic regulatory hormones - regulate glucose, lipid, and energy metabolism. These 2 hormones determine substrate and energy metabolism under different living conditions. Shift of day and night affects the clock system and metabolism probably through altered insulin and GH secretion. Methods: Five-week-old male mice were randomly assigned to a rotating light (RL) group (3-day normal light/dark cycle followed by 4-day reversed light/dark cycle per week) and normal light (NL) group. Body weight and food intake were recorded every week. Series of blood samples were collected for pulsatile $\mathrm{GH}$ analysis, glucose tolerance test, and insulin tolerance test at 9,10 , and 11 weeks from the start of intervention, respectively. Indirect calorimetric measurement was performed, and body composition was tested at 12 weeks. Expressions of energy and substrate metabolism-related genes were evaluated in pituitary and liver tissues at
\end{abstract}

karger@karger.com www.karger.com/nen

Karger $\stackrel{\text { ' }}{5}$

GOPEN ACCESS
C 2021 The Author(s)

Published by S. Karger AG, Basel

This is an Open Access article licensed under the Creative Commons Attribution-NonCommercial-4.0 International License (CC BY-NC) (http://www.karger.com/Services/OpenAccessLicense), applicable to the online version of the article only. Usage and distribution for commercial purposes requires written permission. the end of 12-week intervention. Results: The RL group had an increased number of GH pulsatile bursts and reduced $\mathrm{GH}$ mass/burst. RL also disturbed the GH secretion regularity and mode. It suppressed insulin secretion, which led to a disturbed insulin/GH balance. It was accompanied by the reduced metabolic flexibility and modified gene expression involved in energy balance and substrate metabolism. Indirect calorimeter recording revealed that $\mathrm{RL}$ decreased the respiratory exchange ratio (RER) and oxygen consumption at the dark phase, which resulted in an increase in fat mass and free fatty acid levels in circulation. Conclusion: RL disturbed pulsatile GH secretion and decreased insulin secretion in male mice with significant impairment in energy, substrate metabolism, and body composition.

(C) 2021 The Author(s)

Published by S. Karger AG, Basel

\section{Introduction}

Insulin and GH, 2 vital metabolic hormones, are involved in regulating glucose, lipid, and energy metabolism [1]. GH and insulin produce the opposite influence on substrate metabolism. Insulin reduces the blood glu-
Correspondence to:

Chen Chen, chen.chen@uq.edu.au

Lixin Guo,glx1218@163.com 
cose level and promotes the lipogenesis in tissues, whereas GH increases the circulating glucose level and promotes the lipolysis. As such, a concept of insulin/GH balance has been proposed to reflect substrate and energy metabolism states under diverse physiological and pathophysiological conditions [1]. Insulin/GH balance was proposed to determine the fat accumulation and energy expenditure and may predict the progress of obesity and diabetes $[1,2]$. Our previous research provided strong supporting evidence [3,4], although the detailed mechanism has not been totally illustrated.

Disturbed light/dark cycle always contributes to weight gain, increased hepatic lipid storage, imbalanced secretion of metabolic hormones, and inflammation [5-8]. Metabolic consequence is always accompanied by the expression changes of clock genes $[9,10]$. Specifically, clock gene mutations and disturbed light/dark cycles are associated with insulin resistance and increased morbidity and mortality [11]. Central and peripheral clock genes and clock-controlled genes, such as PPAR $\alpha$ and PGC1 $\alpha$, are also involved in the regulation of metabolic rhythms [12]. Several metabolic regulatory hormones including growth hormone $(\mathrm{GH})$ are secreted with a circadian rhythm. Animal experiments of disturbed light/dark cycle showed different outcomes of GH levels. Constant light or dark decreased the GH secretion [7] and disturbed the pulsatile GH secretion mode [13]. One other study showed that the disturbed light/dark cycle had no effect on random GH secretion [14]. There was no related research to identify the effect of rotating light (RL) on pulsatile secretion of GH. However, an altered light/dark cycle resulted in an increase in random insulin levels [15] or no difference of fasting insulin levels [16]. Different results may be caused by different ages of mice and different intervention periods. There was however a strong interaction between the clock system and the hypothalamic-pituitary-adrenal axis $[17,18]$. GH had similar a pulsatile secretion pattern like glucocorticoids, and there was evidence supporting the link between light and GH secretion $[19,20]$. The GH-IGF-1 axis could be potentially changed by light shifting to affect the peripheral clock system and body metabolism.

In terms of energy metabolism, mice in normal light (NL)-cycle condition have higher respiratory quotient, activity, and energy expenditure during the dark phase than those during the light phase [6]. There is a significant circadian rhythm of food intake under NL cycle condition, whereas there was no obvious difference between the light phase and dark phase under RL conditions [6]. Evidence showed that mice fed with a high-fat diet only during the 12-h light phase gained more weight than mice fed only during the 12-h dark phase [21]. Light-phase feeding caused a decrease in body temperature during the dark phase, which could lead to the increase in fat mass and weight gain [21]. The change of the respiratory exchange ratio (RER) during an overnight fasting was considered to indicate metabolic flexibility between using glucose or fatty acids to produce energy, an important aspect of body energy metabolism. Insulin resistance was a crucial factor to influence metabolic flexibility in obesity $[22,23]$. GH and insulin had a significant effect on metabolic flexibility and energy metabolism $[1,24]$. However, there was not any sufficient evidence to establish the link between the disturbed light/dark cycle and GH profiles on energy metabolism.

This study aimed to identify the influence of RL on 2 important metabolic hormones (insulin and GH) and substrate and energy metabolism in mice. Results indicated that RL changed the secretion profiles of GH and affected substrate and energy metabolism.

\section{Materials and Methods}

Animals

Male mice on a C57BL6/L background were attained from an animal facility in the University of Queensland. All mice were fed under a 12-h light/dark cycle with a room temperature of $21 \pm 2^{\circ} \mathrm{C}$ and a humidity of $34 \pm 4 \%$ for 1 week to adapt to the environment at the animal facility of the Institute for Bioengineering and Nanotechnology, University of Queensland. All the mice had free access to water.

\section{Experimental Design}

To identify the effect of a disturbed light/dark cycle on metabolism in mice, 5 -week-old male mice $(N=20)$ were randomly assigned to 2 groups (10:10) as follows: RL and NL. The NL group was defined as a normal 12:12-h light/dark cycle, with light on at 8 a.m. (ZT0) and light off at 8 p.m. (ZT12). The RL group was defined as a 3 -day normal light/dark cycle followed by a 4 -day reversed light/dark cycle with light on at 8 p.m. (ZT12) and light off at 8 a.m. (ZT0). This RL condition lasted for the entire experiment until the final week. All experiment procedures were performed during the normal light/dark cycle in the RL group.

Body weight and food intake were recorded weekly. Blood collection for ITT, GTT, and GH measurements was performed at 7, 7 , and 8 weeks after the intervention, respectively. Indirect calorimetric recording was performed at 9 weeks after the intervention. At the end of the 10-week period, mice were sacrificed by injecting sodium pentobarbital $(32.5 \mathrm{mg} / \mathrm{mL})$ under light condition. Terminal blood was collected from the heart, and tissues were frozen at $-80^{\circ} \mathrm{C}$ for gene expression measurement.

\section{Indirect Calorimetric Recording}

All mice were fed in the chambers of a TSE Phenomaster (TSE systems, Germany) for 1 week. All mice had a habituation period 


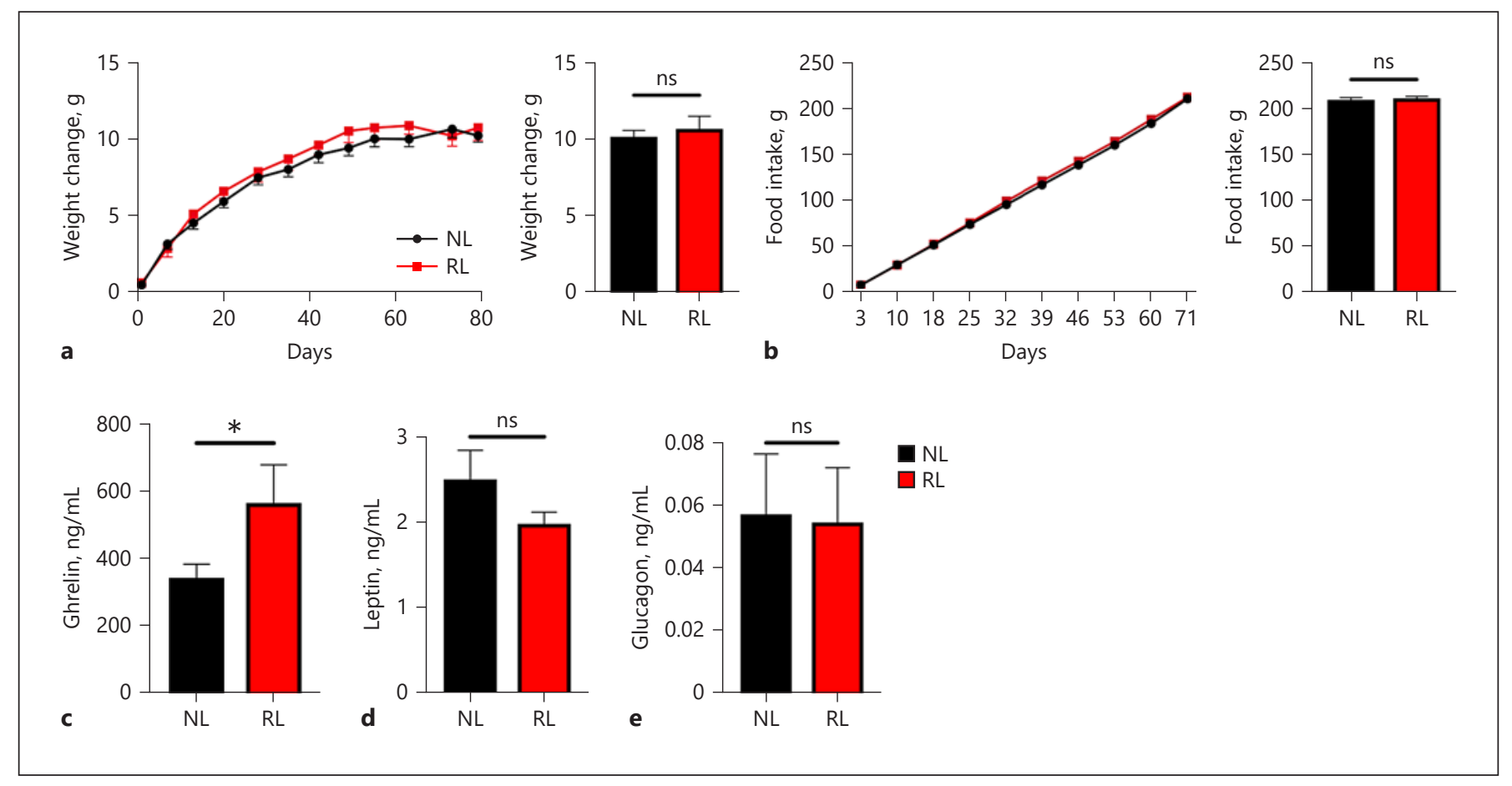

Fig. 1. RL did not affect weight change, food intake, leptin, and glucagon levels but ghrelin levels. Weight change (a) and food intake at the end of the experiment in both groups (b). Circulating ghrelin level (c), leptin level (d), and glucagon level at the end of the experiment in both groups (e). Data are presented as mean \pm SEM, $n=8$ each group. ${ }^{*} p<0.05$; ns, nonsignificance; RL, rotating light; NL, normal light.

for 1 week to individual housing before starting measurements. Body weight, food intake, RER, oxygen consumption, and activity were recorded hourly. To identify the metabolic flexibility, all mice were fasted for $12 \mathrm{~h}$ in the Phenomaster to record data. Body mass and lean mass were calculated by an NMR machine (Bruker, USA).

\section{ITT and GTT}

Mice were fasted for $3 \mathrm{~h}$ and $16 \mathrm{~h}$ before the insulin tolerance test (ITT) and glucose tolerance test (GTT). Insulin (0.3 U/kg, Sigma) was injected intraperitoneally for the ITT, and glucose (2 g/ $\mathrm{mg}$ ) was infused by oral gavage for the GTT. Blood glucose was measured by a glucose ketone meter (Nova Stat Strip Xpress Glucose Hospital Meter, Nova Biomedical, UK) at several time points $(0,15,30,60,90$, and $120 \mathrm{~min}$ for the GTT and $0,15,30,45,60$, and $90 \mathrm{~min}$ for the ITT).

\section{Blood Collection for GH Measurement}

All mice were trained for 4 weeks before the blood collection for $\mathrm{GH}$ testing. According to an established blood collection and analysis method for GH testing [25], $2 \mu \mathrm{L}$ of blood was collected from the tail tip of all mice in a 10-min interval. Then, $58 \mu \mathrm{L} 0.01$ M PBS with $0.05 \%$ Tween 20 and blood were mixed. Blood collection process started at 09:30 a.m. and ended at 3:30 p.m. during the light phase in both groups on the third day under the normal light/ dark schedule. All mixed samples were frozen at $-80^{\circ} \mathrm{C}$ for $\mathrm{GH}$ measurement [25].

Rotating Light Changes Growth Hormone Pulsatile Secretion

\section{Hormones Measurement}

Terminal blood was collected by cardiac puncture under the light condition at the tenth week during the normal light/dark cycle, and the plasma was used for the measurement of several target hormones (acylated ghrelin, glucagon, leptin, and feeding insulin) by the mouse Multiplex kit (BioRad). Fasting insulin and C-peptide levels were measured from the plasma collected from the tail under the light condition during the normal light/dark cycle at the ninth week after the removal of food for $14 \mathrm{~h}$ by the same Multiplex kit (BioRad). Plasma free fatty acid (FFA) concentration was measured from the plasma of the terminal blood samples by the NEFA C kit (Wako).

\section{Genes Expression Levels Measurement}

The total RNA was extracted by the PureLink RNA Mini Kit (Thermo Fisher Scientific) from the pituitary gland and liver. Total RNA was reversed to cDNA by the iScriptTM RT Supermix for quantitative PCR (Bio-Rad). The amplification of cDNA was performed by running SsoAdvancedTM Universal SYBR Green Supermix (BioRad) and QuantStudio 6 Flex Real-time PCR system (Thermo Fisher Scientific). The gene expression compared to the housekeeping gene (beta-actin) was calculated by the $2^{-\Delta \Delta C T}$ method. The sequences of primer pairs are shown in online suppl. Table 1; for all online suppl. material, see www.karger.com/doi/10.1159/000518338. 


\section{Statistics}

Statistical analysis was performed through Graphpad prism 8. Student's $t$ test was performed to compare between 2 groups. Data not meeting the criteria for normal distribution were analyzed using the nonparametric Mann-Whitney test. Bonferroni's method was used to adjust $p$ value to accommodate multiple tests if multiple comparisons were not independent of each other. Mean \pm
SEM was used to express all the results with ${ }^{*} p<0.05,{ }^{* *} p<0.01$, $* * * p<0.001$. The results were considered statistically significant when $p<0.05$. Deconvolution analysis was performed by an established method [25] to identify the GH secretion profile with data clearance. The insulin/GH ratio was calculated by Pulse XP software (version 2.002).
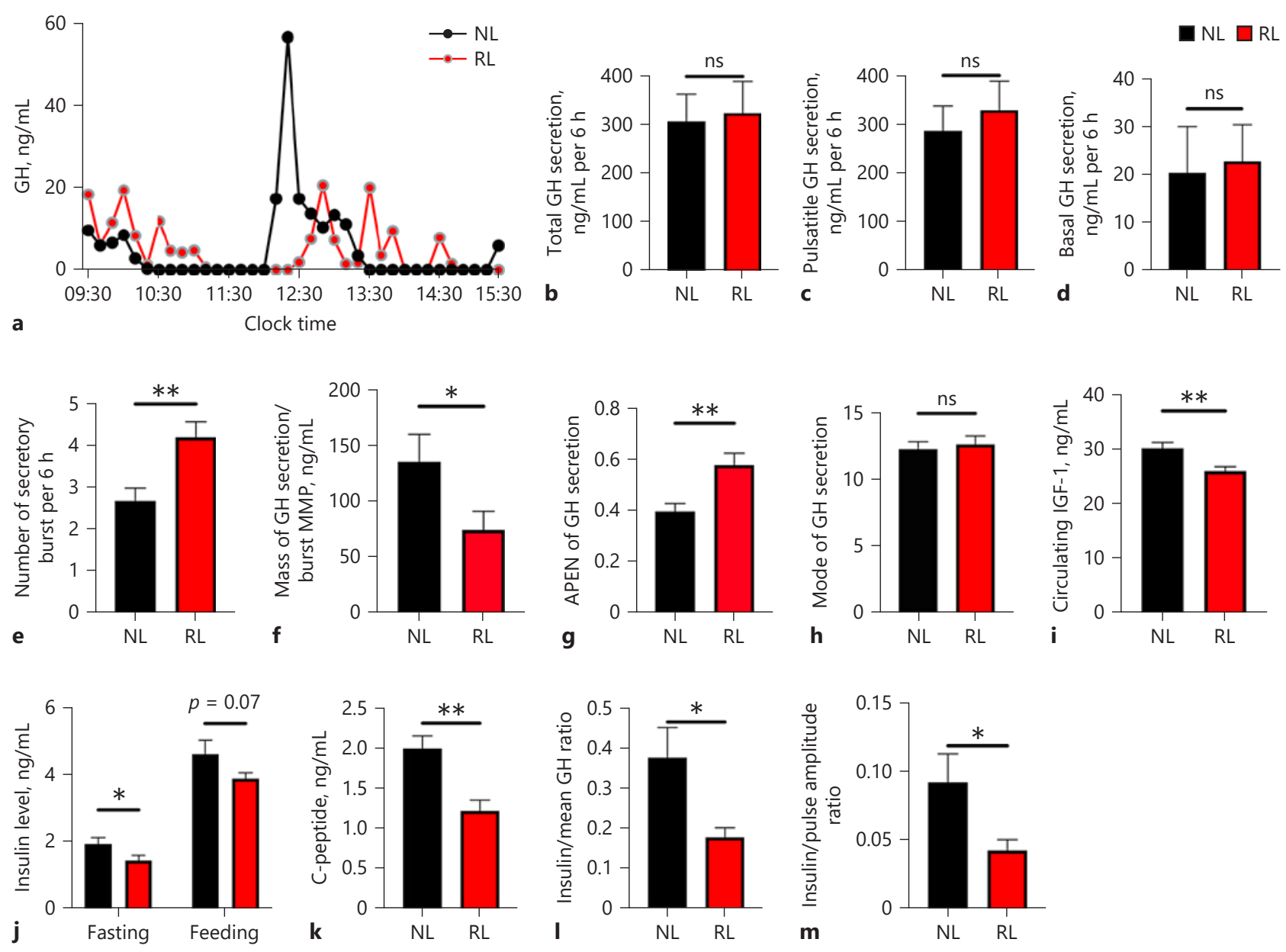

Fig. 2. RL decreases insulin release and disturbed pulsatile GH secretion rhythm in WT mice. a Representative GH secretion examples in both groups during a 6-h testing period. $\mathbf{b}-\mathbf{h}$ Deconvolution analysis results showed the changes of total GH secretion (b), pulsatile GH secretion (c), basal GH secretion (d), number of burst (e), mass of GH secretion/burst (f), APEN of GH secretion (g), mode of GH secretion (h). i Circulating IGF-1 level. j Fasting and feeding insulin levels. k Feeding C-peptide level. I Insulin/mean GH concentration ratio in both groups. $\mathbf{m}$ Insulin/GH pulse amplitude ratio in both groups. Data are presented as mean \pm SEM, $n=10$ each group. ${ }^{*} p<0.05,{ }^{* *} p<0.01$; ns, nonsignificance; APEN, approximate entropy; RL, rotating light; NL, normal light; $\mathrm{GH}$, growth hormone.

Fig. 3. RL decreased energy expenditure and increased fat mass in male WT mice. RER (a), oxygen consumption (b), activity (c) and food intake were recorded at light and dark phase in both groups (d). Fat mass and lean mass were tested in both groups (e). Data are presented as mean \pm SEM, $n=8$ each group. ${ }^{*} p<0.05,{ }^{* *} p<0.01,{ }^{* * *} p$ $<0.001$; ns, nonsignificance; RER, respiratory exchange ratio; RL, rotating light; NL, normal light.

(For figure see next page.) 

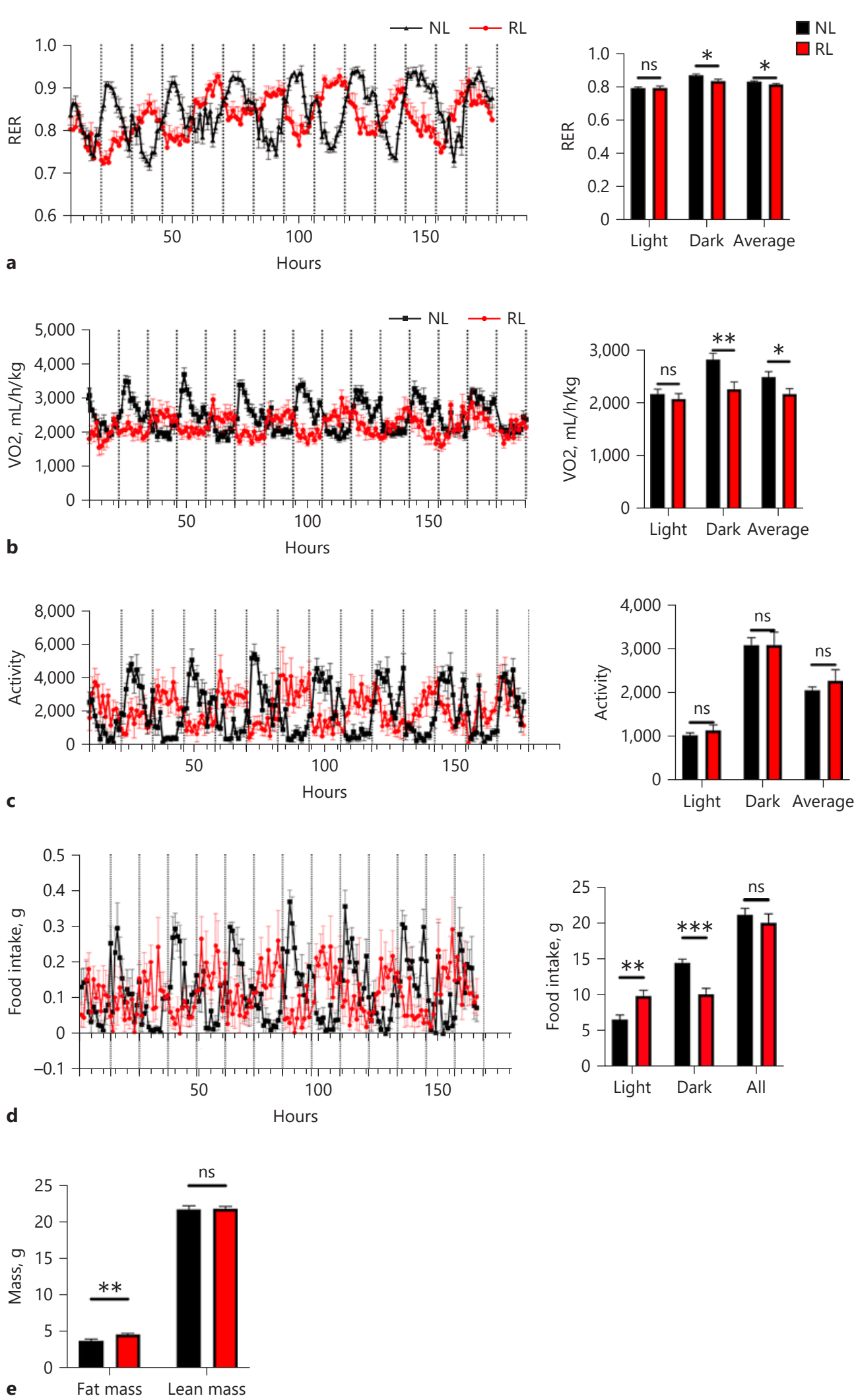

3 


\section{Results}

Ten-Week RL Had No Effect on Weight Change and Food Intake but Acylated Ghrelin Levels

To identify the effect of RL on metabolism and related hormones, we measured the weight change and food intake of mice each week. We also tested the circulating ghrelin, leptin, and glucagon levels at the end of experiment in both groups. Results indicated that there was no significant difference in weight change (Fig. 1a), food intake (Fig. 1b), leptin level (Fig. 1d), and glucagon level (Fig. 1e) in both groups. However, RL increased the acylated ghrelin level (Fig. 1c), which could disturb the rhythm of food intake as described in the following energy balance experiment (Fig. 3d).

\section{RL Altered Insulin Level and GH Profile in WT Mice}

As we described before, pulsatile GH secretion decreased significantly when facing challenges like fasting [26]. To better identify the effect of RL, we tested the GH profile under a fed state. Deconvolution analysis results showed the changes of total GH secretion (Fig. 2b), pulsatile GH secretion (Fig. 2c), basal GH secretion (Fig. 2d), number of burst (Fig. 2e), mass/burst (Fig. 2f), approximate entropy (Fig. 2g), and mode (Fig. 2h). Although there was no significant difference in total $\mathrm{GH}$, pulsatile $\mathrm{GH}$, and basal GH secretion between 2 groups, the RL group had an increased number of bursts and reduced mass/burst. RL also disturbed the GH secretion regularity and mode (Fig. 2g, h). Insulin-like growth factor 1 (IGF-1) was considered as a biomarker for the downstream pathways of the $\mathrm{GH}$ receptor (GHR). Circulating IGF-1 measurement (Fig. 2i) confirmed that RL reduced IGF-1 levels and disturbed the downstream pathway of $\mathrm{GH}$ by regulating the $\mathrm{GH}$ secretion mode. We tested the plasma insulin level in fasting and feeding states; while the fasting insulin level reflected basal insulin secretion, the feeding insulin level reflected beta-cell function in response to glucose increase after meal. Our results showed that RL decreased the fasting insulin level (Fig. 2j), Cpeptide level, (Fig. 2k) and tended to reduce the feeding insulin level (Fig. 2j). As the plasma-circulating insulin/ GH ratio was associated with energy metabolism [1], the insulin/mean GH concentration ratio (Fig. 2l) and insulin/GH pulse amplitude ratio (Fig. $2 \mathrm{~m}$ ) were measured and calculated in both groups. Results showed that RL significantly disturbed the insulin/GH ratio which may be associated with the changes in energy expenditure and fat accumulation.
RL Disturbed Energy Balance and Increased Fat Mass in Mice

To determine the effect of RL on phenotypic changes, body composition and calorimeters were recorded in both groups. Indirect calorimeter recording revealed that RL decreased the RER in the dark phase, with no difference in the light phase (Fig. 3a), reflecting a dominant consumption of fatty acids over carbohydrates in the dark phase. RL also decreased the oxygen consumption at the dark phase with no difference in the light phase (Fig. 3b), which may contribute to increased fat mass (Fig. 3e). RL also disturbed the feeding rhythm by increasing the food intake in the light phase and decreasing it in the dark phase (Fig. 3d). This disturbed feeding pattern may explain the changes of the RER and oxygen consumption upon RL. There was no significant difference in activity either at the light or dark phase between 2 groups (Fig. 3c). To determine specific changes of energy metabolism in the whole week upon RL, we analyzed every day's energy recording including RER, oxygen consumption, activity, and food intake (online suppl. Fig. 1). Results showed that oxygen consumption decreased significantly in the first day and the fourth day (the day of switching light condition) (online suppl. Fig. 1a); RER and food intake decreased significantly in the first day (online suppl. Fig. 1b, d) upon RL, whereas there was no significant difference in the remaining days. There was no significant difference of activity (online suppl. Fig. 1c) between 2 groups which was consistent with the results showed in Figure 3. Although results revealed significant difference of food intake in the first day, there was no difference of total food intake between 2 groups. All the data may indicate that RL mainly affects the energy metabolism in the first day of changing light.

\section{RL Reduced Metabolic Flexibility and Increased the \\ Blood FFA Level}

To identify the effect of RL on metabolic flexibility upon fasting challenge, we recorded the energy metabolism for $12 \mathrm{~h}$ during the fasting state. Results showed that the RER did not decrease rapidly upon RL which indicated a dominant consumption of carbohydrates over fat during the transition state (Fig. 4a). This suggested that $\mathrm{RL}$ disturbed the normal metabolic flexibility under a normal light/dark cycle. At the same time, RL decreased oxygen consumption (Fig. 4b) and tended to reduce total activity (Fig. 4c) during the fasting period, which contributed to a weight loss upon fasting challenge. Insulin resistance was usually related to the metabolic inflexibility and parallel with defects in FFA metabolism [23]. We then
Wang/Huang/Huang/Tan/Chen/ Roelfsema/Chen/Guo 
Fig. 4. RL reduced metabolic flexibility and increased FFA level. a-c RER (a), oxygen consumption (b), and activity were recorded during fasting period (20:00 h to $08: 00 \mathrm{~h}$ next day) in 1-h intervals (c). d ITT in both groups. e FFA levels at the end of experiment in both groups. Data are presented as mean \pm SEM, $n=5-7$ each group. ${ }^{*} p<0.05$, ${ }^{* *} p<0.01$; ns, nonsignificance; RL, rotating light; FFA, free fatty acid; ITT, insulin tolerance test; RER, respiratory exchange ratio; NL, normal light.
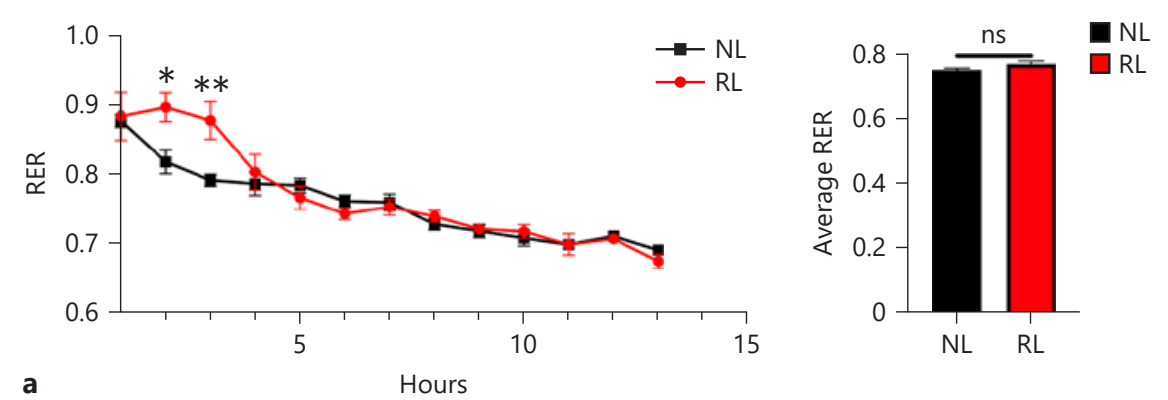

a

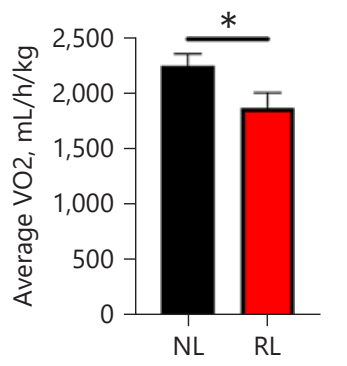

b
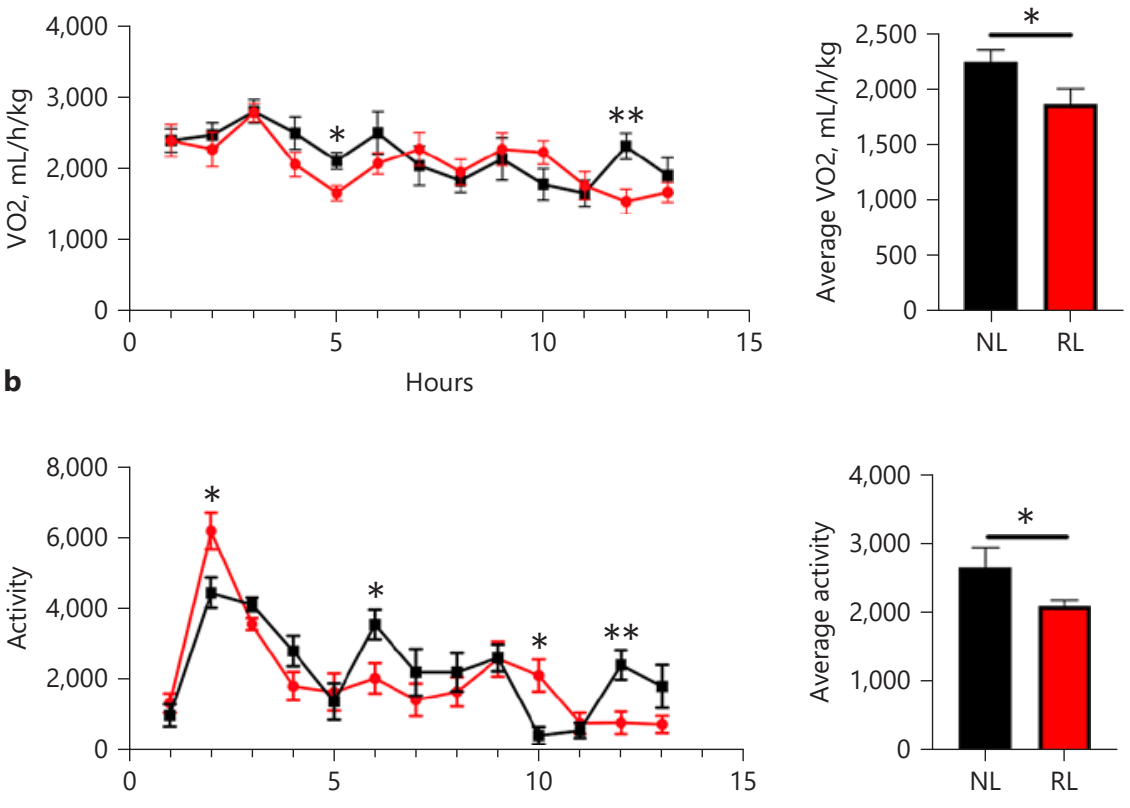

C
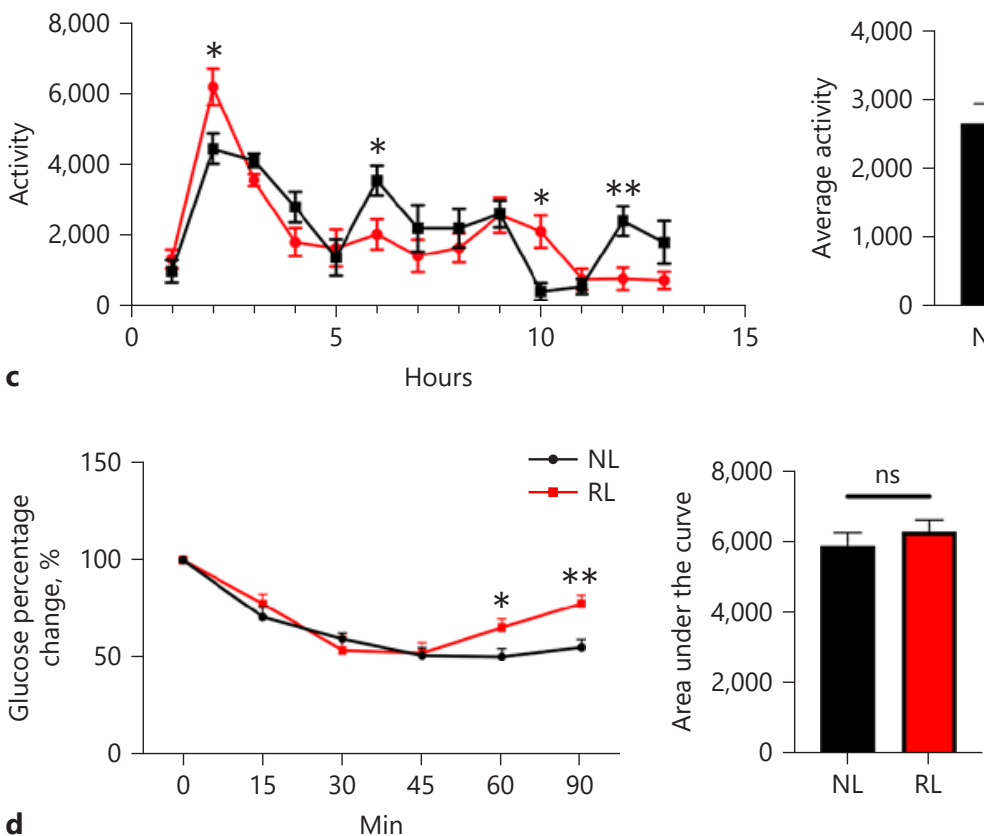

d

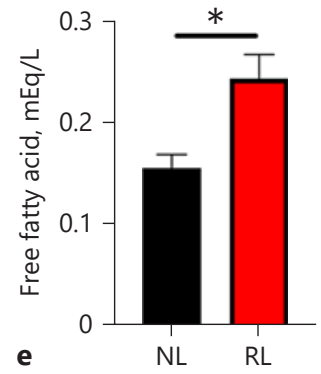




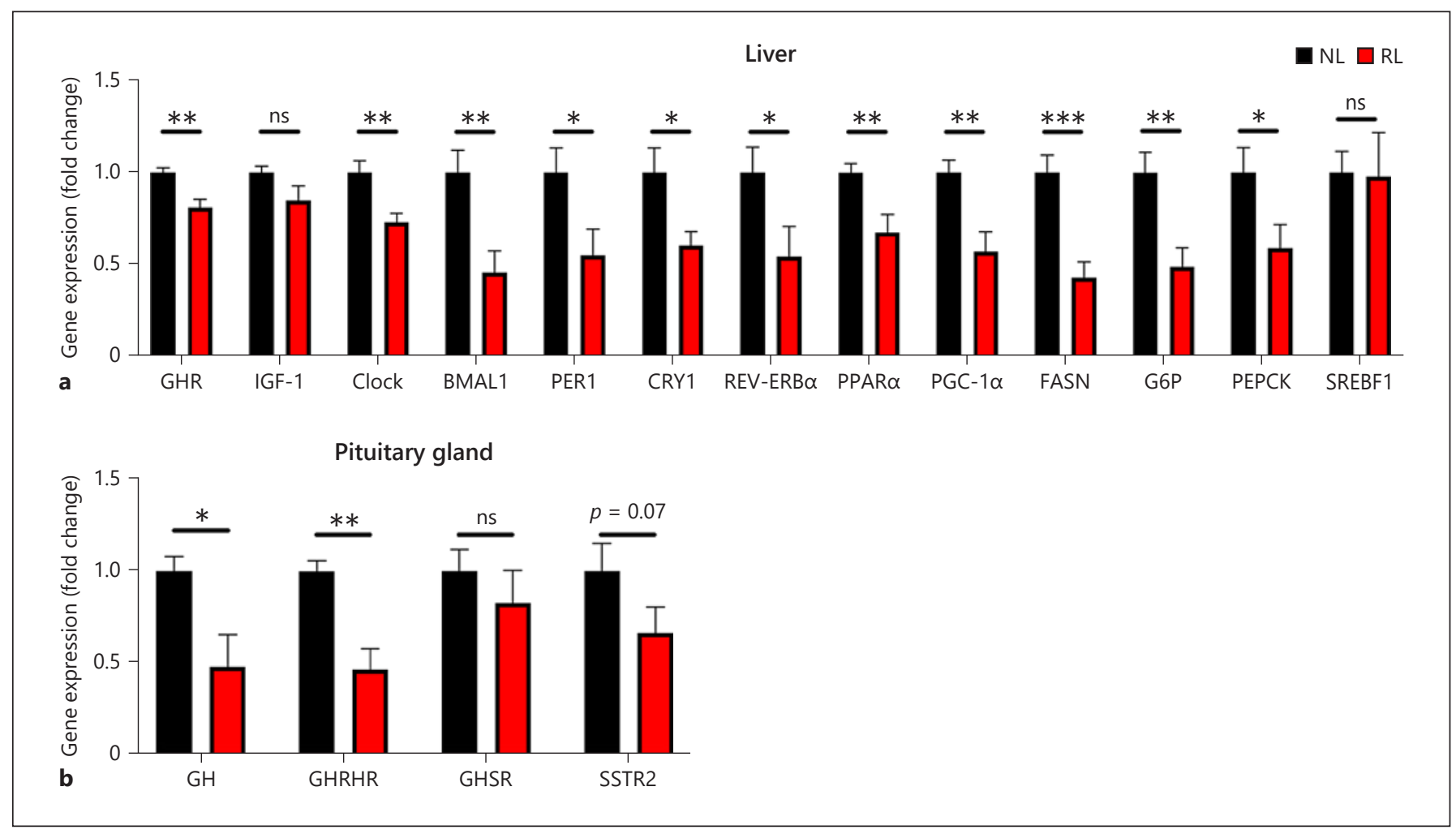

Fig. 5. RL decreased genes expression involved in GHR signaling in pituitary and clock systems and substrate metabolism in liver. Gene expression fold change was calculated by qPCR in liver (a) and pituitary gland (b). Data are presented as mean \pm SEM, $n=8$ each group. ${ }^{*} p<0.05,{ }^{* *} p<0.01,{ }^{* * *} p<0.001$; ns, nonsignificance; GHR, GH receptor; RL, rotating light; qPCR, quantitative PCR; NL, normal light.

performed the ITT and measured blood FFAs in both groups (Fig. 4d, e). Although there was no significant difference in the ITT between the 2 groups, RL increased the circulating level of FFAs. Early stage of RL here might be insufficient to lead to insulin resistance yet.

\section{$R L$ Disturbed Expression of Genes Involved in GHR Signaling and Substrate Metabolism in the Pituitary Gland and Liver}

To determine the effects of RL at molecular level, we tested metabolism-related gene expression in the pituitary gland and liver. GHR and IGF-1 are often regarded as the downstream signaling targets of GH. RL clearly decreased the expression of gene GHR (Fig. 5a), which affirmed the impact of disturbed pulsatile GH secretion mode. However, there was no significant expression difference of gene IGF-1 between 2 groups. At the same time, expressions of GH-related genes were measured in the pituitary gland (Fig. 5b). RL significantly reduced the expressions of GH and GHRH receptor in pituitary, which also affirmed the impact of light on GH. However, there was no obvious difference in expression of GHSR and SSTR2 between the 2 groups. Expression changes of clock genes (CLOCK, BMAL1, PER1, CRY1, and REV$E R B a$ ) proved that circadian processes were at work in response to RL (Fig. 5a). RL also reduced the expression of genes involved in lipid oxidation (PPAR $\alpha$ ), lipogenesis (FASN), and gluconeogenesis (G6P, PEPCK, and PGC$1 \alpha$ ) in the liver (Fig. 6a). There was no significant expression difference on the lipogenic gene SREBF1 between the 2 groups.

\section{RL Disturbed Glucose Metabolism}

To identify the effect of RL on glucose metabolism, we performed GTT in both groups. Interestingly, RL improved glucose tolerance compared with the normal light/dark cycle (Fig. 6a) which may be due to changed clock gene expression [27]. However, there was an increased fasting glucose level upon RL, with no difference in the feeding glucose level (Fig. 6b). 


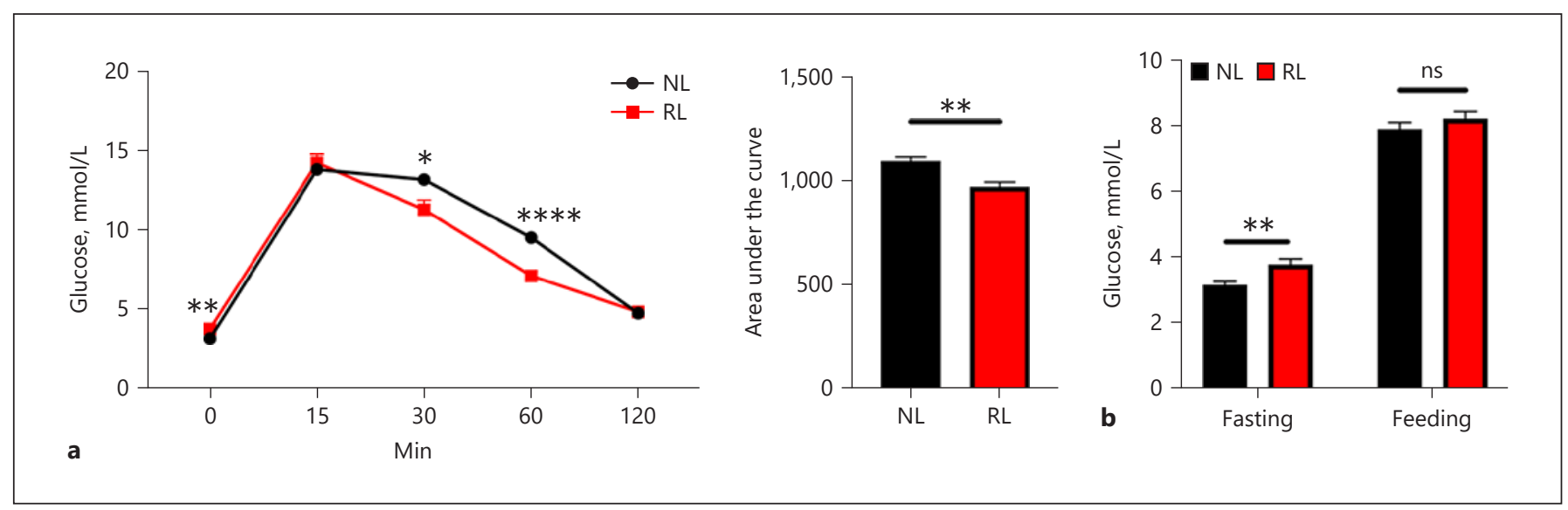

Fig. 6. RL disturbed glucose tolerance. a Glucose tolerance test in both groups. $\mathbf{b}$ Fasting and feeding glucose levels in both groups. Data are presented as mean \pm SEM, $n=10$ each group. ${ }^{*} p<0.05,{ }^{* *} p<0.01$; ns, nonsignificance; RL, rotating light; NL, normal light.

\section{Discussion}

Insulin-GH balance is recently considered as a significant part involved in substrate and energy metabolism [1, 28]. GH and insulin have the opposite influence on substrate metabolism. Insulin reduces the blood glucose level and promotes the lipogenesis, whereas GH increases the circulating glucose level and promotes the lipolysis. While many factors or interventions had been well established to effectively alter insulin or GH levels separately, interaction of 2 hormones has not been thoroughly investigated. Diverse light conditions like constant light or constant dark had a significant effect on rhythm release of GH [13, 20, 29]. Liver-specific deletion of GHR indicated an important effect of GH pathways in hepatic lipid metabolism [30]. The specific mechanism was not clarified yet. In terms of the effect of diverse light conditions on insulin levels, disturbed light condition reduced the insulin level upon normal chow, whereas increased it upon high-fat diet [31]. However, another research study revealed that chronic light cycle disruption did not change insulin levels [16]. Our results revealed that RL disturbed rhythm GH release model and insulin/GH balance. The expression of GH-related genes (GH, GHRH receptor, and GHR) in the pituitary gland and liver, and the circulating IGF-1 level also confirmed this hypothesis. Our results showed that RL decreased the insulin levels which may be due to the changes of the clock components [27]. A disturbed clock system may contribute to a disturbed rhythm of glucose tolerance [27]. Although the change of insulin/GH balance in this project (decreased insulin and
GH levels) was not the same extend and direction as the most common change observed in hyperphagia and obesity (increased insulin and decreased GH levels), such change of insulin/GH balance in this experiment may still contribute to the increased fat mass and plasma FFAs. This could be a potential lifestyle influence on body metabolism.

The circadian rhythm is determined by the activation of the hypothalamic suprachiasmatic nucleus (SCN), which is entrained by light [18]. For instance, there was a strong interaction between the clock system and the hypothalamic-pituitary-adrenal axis $[17,18]$. The circadian clock system is divided into the following 2 core classes: the central clock system located in the SCN and the peripheral clock system located in peripheral organs such as the liver $[32,33]$. However, neurohumoral signals from the SCN may regulate the activity of the hypothalamus and affect the pulsatile secretion of pituitary hormones including GH [33]. Hence, several hormones secreted by the pituitary gland have a clear circadian rhythm. The rhythmic expression of clock genes was also associated with energy metabolism and substrate metabolism in liver [34]. It is therefore assumed that there is a significant link between rhythmic release GH and rhythmic expression of peripheral clock genes. Clinical trial performed in hypopituitary patients confirmed that $\mathrm{GH}$ regulated the expression of clock genes in muscle [35]. The gene expression change of GHR and circulating change of IGF-1 was caused by the GH secretion change in this experiment. The expression changes of clock genes (Clock, Bmal1, Per1, Cry1, and Rev-erba) in the liver suggested 
that circadian processes were responding to RL. GH may be a target of RL to further regulate the peripheral clock system. The detailed relationship among light, pulsatile secretion of $\mathrm{GH}$, and clock system remained to be clarified.

Changes in energy expenditure and fat mass are related to insulin/GH balance in diverse disease conditions [1]. Several clinical trials reported that blocking GHR contributed to decreased basal energy expenditure, which confirmed the effect of GH on energy metabolism [36, 37]. Results in this experiment revealed that RL decreased RER and oxygen consumption in the dark phase without affecting total food intake. Disturbed feeding pattern upon RL may cause the changes of RER and oxygen consumption in the dark phase, which lead to an increase in fat mass [38]. The mechanism however remains unclear. Changes of the $\mathrm{GH}$ secretion mode and regularity upon RL may contribute to disturbed energy metabolism. The decreased RER in the dark phase signified more fat consumption which was at least partially due to decreased food intake in the dark phase. Although there was no significant difference in total food intake between 2 groups, an increased ghrelin level and disturbed food intake rhythm could be related to disturbed body metabolism. At the same time, oxygen consumption affected energy expenditure more than RER change, which may lead to increased fat mass upon RL. These changes of energy metabolism only occurred in the first day of the light shift, which indicated that long-term metabolic influence of shift workers was caused by an accumulated damage from the first switching day.

Fuel selection, during the fasting to feeding period, is considered as a core part of metabolic flexibility [23]. Metabolic flexibility was evaluated and reported through the change in the RER from fasting to feeding state [23]. In this experiment, the RER decreased slowly compared to that in the control group upon RL, which indicated a dominant consumption of carbohydrates over fatty acids during the feeding to fasting transition state. This proved that $\mathrm{RL}$ disturbed the metabolic flexibility compared with that under a normal light/dark cycle. Fatty acids were the primary energy source during this transition state. Increased blood FFAs upon RL also proved the reduced metabolic flexibility. It is unclear whether the change of FFAs is caused by decreased metabolic flexibility. It may also be caused by disturbed circadian control of metabolism. The GH/IGF-1 axis is often involved in fatty acid metabolism and insulin resistance [39-41]. Fat oxidation was stimulated by $\mathrm{GH}$, and circulating FFAs were consumed as a major energy source [40]. However, insulin levels decreased significantly, which promoted lipolysis. In terms of insulin resistance, the results showed no significant difference between 2 groups, which might be due to insufficient intervention time. Normalized data of glucose were chosen to reflect the insulin resistance because of lower fasting glucose in the RL group, which might be due to less food intake prior to 3 - $h$ fasting. The elevated glucose levels at 45 and 90 min upon RL may suggest an altered action of counter-regulatory hormones (glucagon, corticosterone, and GH). In summary, RL may diminish metabolic flexibility through the GH/ IGF-1 axis.

$\mathrm{GH}$ also plays a crucial role in substrate metabolism in the liver. Recent studies revealed that GHR, CD36, and PPARa participated in this process [42-44], which was in line with our results. GH promoted gluconeogenesis in the liver [24], partly by activating the STAT5 pathway, which increased the expression of G6P and PEPCK [45]. Interestingly, RL increased the fasting glucose level but improved glucose tolerance. Evidence showed that the whole-body inactivation of BMAL1 or CLOCK contributed to suppressed glucose rhythm and impaired gluconeogenesis $[46,47]$. This circadian misalignment altered the glucose tolerance rhythm, whereas the specific mechanism still remained unclear [47]. $\mathrm{GH}$ regulated the marker of lipid oxidation (PGC-1 $\alpha$ ) [42], which suggested the relationship between GH and hepatic lipid metabolism. There was a possibility that the peripheral clock system was a target of GH to regulate the substrate and lipid metabolism in the liver [29]. PGC- $1 \alpha$ bound to BMAL1 and PPAR bound to PER2 in the liver to regulate lipid and glucose homeostasis [33]. Interactions among $\mathrm{GH}$, peripheral clock system, and metabolism were not fully clarified and required further investigation.

\section{Conclusions}

In summary, this research revealed that the RL regulated insulin and GH secretion to influence the body substrate/energy metabolism.

\section{Statement of Ethics}

The research presented in the article was ethically performed in accordance with institutional guidelines based on National Institutes of Health standards and the procedures of the animal experiment were approved by the Ethics Committee of the University of Queensland (approval number SBMS/500/18). 


\section{Conflict of Interest Statement}

There are no conflicts of interest with any of the authors.

\section{Funding Sources}

This work was supported by the National Health and Medical Research Council grant of Australia, University of Queensland, Australia, and the National Natural Science Foundation of China (Grant Nos. 81670763 and 81471050).

\section{Author Contributions}

Weihao Wang, Zhengxiang Huang, Lili Huang, Lixin Guo, and Chen Chen designed the experiments. Weihao Wang, Zhengxiang Huang, and Chunlu Tan performed the animal experiments. Weihao Wang and Wanlin Chen performed the ELISA and quantitative PCR analysis. Ferdinand Roelfsema helped analyze the GH data. Weihao Wang prepared the manuscript and figures. Lili Huang, Lixin Guo, and Chen Chen revised the manuscript. All the authors approved the final article.

\section{Data Availability Statement}

All data generated or analyzed during this study are included in this article and its online suppl. material files. Further inquiries can be directed to the corresponding author.

\section{References}

1 Huang Z, Huang L, Waters MJ, Chen C. Insulin and growth hormone balance: implications for obesity. Trends Endocrinol Metab. 2020;31:642.

2 Huang L, Huang Z, Chen C. Rhythmic growth hormone secretion in physiological and pathological conditions: lessons from rodent studies. Mol Cell Endocrinol. 2019;498(September): 110575 .

3 Tan HY, Steyn FJ, Huang L, Cowley M, Veldhuis JD, Chen C. Hyperphagia in male melanocortin 4 receptor deficient mice promotes growth independently of growth hormone. J Physiol. 2016;594(24):7309-26.

4 Huang Z, Huang L, Wang C, Zhu S, Qi X, Chen $Y$, et al. Dapagliflozin restores insulin and growth hormone secretion in obese mice. J Endocrinol. 2020;245(1):1-12.

5 Tsai LL, Tsai YC, Hwang K, Huang YW, Tzeng JE, Tsai Y, et al. Repeated light-dark shifts speed up body weight gain in male F344 rats. Am J Physiol Endocrinol Metab. 2005;289: E212-7.

6 Christie S, Vincent AD, Li H, Frisby CL, Kentish SJ, O’Rielly R, et al. A rotating light cycle promotes weight gain and hepatic lipid storage in mice. Am J Physiol Gastrointest Liver Physiol. 2018;315(6):G932-42.

7 Relkin R. Effects of pinealectomy, constant light and darkness on growth hormone levels in the pituitary and plasma of the rat. J Endocri. 1971;53:289-93.

8 Kim SM, Neuendorff N, Alaniz RC, Sun Y, Chapkin RS, Earnest DJ. Shift work cycle-induced alterations of circadian rhythms potentiate the effects of high-fat diet on inflammation and metabolism. FASEB J. 2018;32(6) 3085-95.

9 Fonken LK, Nelson RJ. The effects of light at night on circadian clocks and metabolism. Endocr Rev. 2014(June):1-25.

10 Arendt J. Shift work: coping with the biological clock. Occup Med. 2010;60(1):10-20.
11 Stenvers DJ, Scheer FAJL, Schrauwen P, la Fleur SE, Kalsbeek A. Circadian clocks and insulin resistance. Nat Rev Endocrinol. 2019; 15(2):75-89.

12 Mazzoccoli G, Pazienza V, Vinciguerra M. Clock genes and clock-controlled genes in the regulation of metabolic rhythms. Chronobiol Int. 2012;29(3):227-51.

13 Tannenbaum GS, Martin JB. Evidence for an endogenous ultradian rhythm governing growth hormone secretion in the rat. Endocrinology. 1976;98(3):562-70.

14 Mustonen AM, Nieminen P, Hyvärinen $\mathrm{H}$. Preliminary evidence that pharmacologic melatonin treatment decreases rat ghrelin levels. Endocrine. 2001;16(1):43-6.

15 Karatsoreos IN, Bhagat S, Bloss EB, Morrison $\mathrm{JH}$, McEwen BS. Disruption of circadian clocks has ramifications for metabolism, brain, and behavior. Proc Natl Acad Sci U S A. 2011;108(4):1657-62.

16 Skinner NJ, Rizwan MZ, Grattan DR, Tups A. Chronic light cycle disruption alters central insulin and leptin signaling as well as metabolic markers in male mice. Endocrinology. 2019;160(October):2257-70.

17 Nader N, Chrousos GP, Kino T. Interactions of the circadian CLOCK system and the HPA axis. Trends Endocrinol Metab. 2010;21(5): 277-86.

18 Nicolaides NC, Charmandari E, Chrousos GP, Kino T. Circadian endocrine rhythms: the hypothalamic-pituitary - adrenal axis and its actions. Ann N Y Acad Sci. 2014;1318: 71-80.

19 Kim JH, White SL, Devlin RH. Interaction of growth hormone overexpression and nutritional status on pituitary gland clock gene expression in coho salmon, Oncorhynchus kisutch. Chronobiol Int. 2015;32(1):113-27.

20 Relkin R. Effects of pinealectomy, constan light and darkness on growth hormone levels in the pituitary and plasma of the rat. J Endocrinol. 1972 May;53(2):28993.
21 Arble DM, Bass J, Laposky AD, Vitaterna $\mathrm{MH}$, Turek FW. Circadian timing of food intake contributes to weight gain. Obesity. 2009; 17(11):2100-2.

22 Galgani JE, Moro C, Ravussin E. Metabolic flexibility and insulin resistance. Am J Physiol Endocrinol Metab. 2008;295(5):E1009-17.

23 Goodpaster BH, Sparks LM. Metabolic flexibility in health and disease. Cell Metab. 2017; 25(5):1027-36

24 Moøller N, Joørgensen JOL. Effects of growth hormone on glucose, lipid, and protein metabolism in human subjects. Endocr Rev. 2009;30(2):152-77.

25 Steyn FJ, Huang L, Ngo ST, Leong JW, Tan HY, Xie TY, et al. Development of a method for the determination of pulsatile growth hormone secretion in mice. Endocrinology. 2011; 152(8):3165-71.

26 Steyn FJ, Leong JW, Huang L, Tan HY, Xie TY, Nelson C, et al. GH does not modulate the early fasting-induced release of free fatty acids in mice. Endocrinology. 2012;153(1):273-82.

27 Marcheva B, Ramsey KM, Buhr ED, Kobayas hi Y, Su H, Ko CH, et al. Disruption of the clock components CLOCK and BMAL1 leads to hypoinsulinaemia and diabetes. Nature. 2010;466(7306):627-31

28 Huang L, Huang Z, Chen C. Rhythmic growth hormone secretion in physiological and pathological conditions: lessons from rodent studies. Mol Cell Endocrinol. 2019;498(June): 110575.

29 Zarrinpar A, Chaix A, Panda S. Daily eating patterns and their impact on health and disease. Trends Endocrinol Metab. 2016;27(2): 69-83.

30 Fan Y, Menon RK, Cohen P, Hwang D, Clemens T, Douglas J, et al. Liver-specific deletion of the growth hormone receptor reveals essential role of growth hormone signaling in hepatic lipid metabolism. J Biol Chem. 2009 Jul 24;284(30):1993744. 
31 Coomans CP, Van Den Berg SA, Houben T, Van Klinken JB, Van Den Berg R, Pronk AC, et al. Detrimental effects of constant light exposure and high-fat diet on circadian energy metabolism and insulin sensitivity. FASEB J. 2013;27(4):1721-32.

32 Gamble KL, Berry R, Frank SJ, Young ME. Circadian clock control of endocrine factors. Nat Rev Endocrinol. 2014;10(8):466-75.

33 Albrecht U. Timing to perfection: the biology of central and peripheral circadian clocks. Neuron. 2012;74(2):246-60.

34 Panda S, Antoch MP, Miller BH, Su AI, Schook AB, Straume M, et al. Coordinated transcription of key pathways in the mouse by the circadian clock. Cell. 2002;109(3):307-20.

35 Sjögren K, Leung KC, Kaplan W, GardinerGarden M, Gibney J, Ho KK. Growth hormone regulation of metabolic gene expression in muscle: a microarray study in hypopituitary men. Am J Physiol Endocrinol Metab. 2007;293(1):E364-71.

36 Moller L, Norrelund H, Jessen N, Flyvbjerg A, Pedersen SB, Gaylinn BD, et al. Impact of growth hormone receptor blockade on substrate metabolism during fasting in healthy subjects. J Clin Endocrinol Metab. 2009; 94(11):4524-32.

37 Weiss EJ, Lee AP, Mulligan K, Schambelan M, Murphy EJ. Growth hormone receptor antag- onism with pegvisomant in insulin resistant non-diabetic men: a phase II pilot study. F1000Res. 2017;6(0):1-11.

38 Fonken LK, Workman JL, Walton JC, Weil ZM, Morris JS, Haim A, et al. Light at night increases body mass by shifting the time of food intake. Proc Natl Acad Sci U S A. 2010; 107(43):18664-9.

39 Moran A, Jacobs DR, Steinberger J, Cohen P, Hong CP, Prineas R, et al. Association between the insulin resistance of puberty and the insulin-like growth factor-I/growth hormone axis. J Clin Endocrinol Metab. 2002; 87(10):4817-20.

40 Krag MB, Gormsen LC, Guo Z, Christiansen JS, Jensen MD, Nielsen S, et al. Growth hormone-induced insulin resistance is associated with increased intramyocellular triglyceride content but unaltered VLDL-triglyceride kinetics. Am J Physiol Endocrinol Metab. 2007; 292(3):E920-7.

41 Quabbe HJ, Bratzke HJ, Siegers U, Elban K. Studies on the relationship between plasma free fatty acids and growth hormone secretion in man. J Clin Invest. 1972;51(9):238898.

42 Liu Z, Cordoba-Chacon J, Kineman RD, Cronstein BN, Muzumdar R, Gong Z, et al. Growth hormone control of hepatic lipid metabolism. Diabetes. 2016;65(12):3598-609.
43 Chhabra Y, Nelson CN, Plescher M, Barclay JL, Smith AG, Andrikopoulos S, et al. Loss of growth hormone-mediated signal transducer and activator of transcription 5 (STAT5) signaling in mice results in insulin sensitivity with obesity. FASEB J. 2019;33(5):6412-30.

44 Sos BC, Harris C, Nordstrom SM, Tran JL, Balázs M, Caplazi $\mathrm{P}$, et al. Abrogation of growth hormone secretion rescues fatty liver in mice with hepatocyte-specific deletion of JAK2. J Clin Invest. 2011;121(4):1412-23.

45 Kim YD, Li T, Ahn SW, Kim DK, Lee JM, Hwang SL, et al. Orphan nuclear receptor small heterodimer partner negatively regulates growth hormone-mediated induction of hepatic gluconeogenesis through inhibition of signal transducer and activator of transcription 5 (STAT5) transactivation. J Biol Chem. 2012;287(44):37098-108.

46 Rudic RD, Mcnamara P, Curtis AM, Boston RC, Panda S, Hogenesch JB, et al. BMAL1 and CLOCK, two essential components of the circadian clock, are involved in glucose homeostasis. PLoS Biol. 2004;2(11):e377.

47 Zhong LX, Li XN, Yang GY, Zhang X, Li WX Zhang QQ, et al. Circadian misalignment alters insulin sensitivity during the light phase and shifts glucose tolerance rhythms in female mice. PLoS One. 2019;14(12):e022581316. 